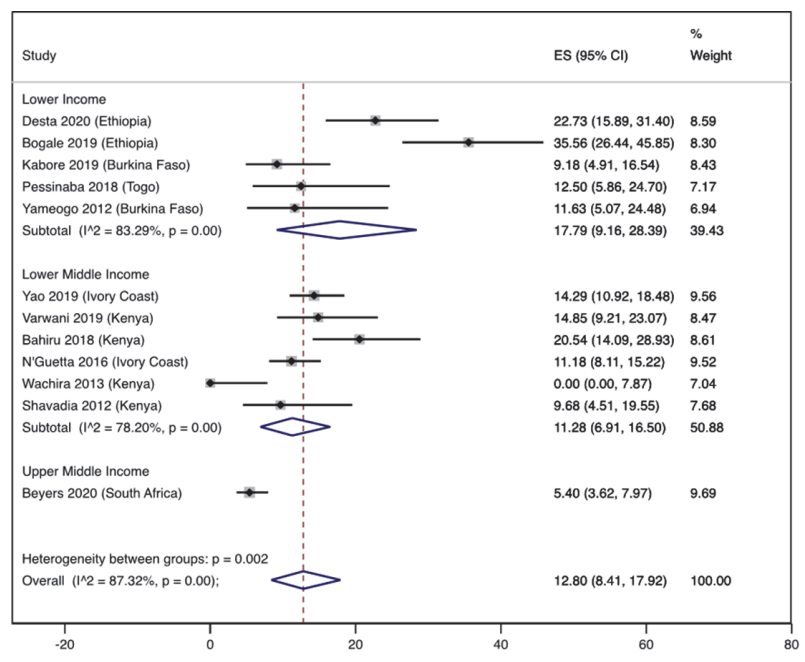

Abstract 43 Figure 1 In hospital mortality of patients with STEMI stratified by income level of country. Effect Size (ES) is the \% of patients with STEMI who died in hospital

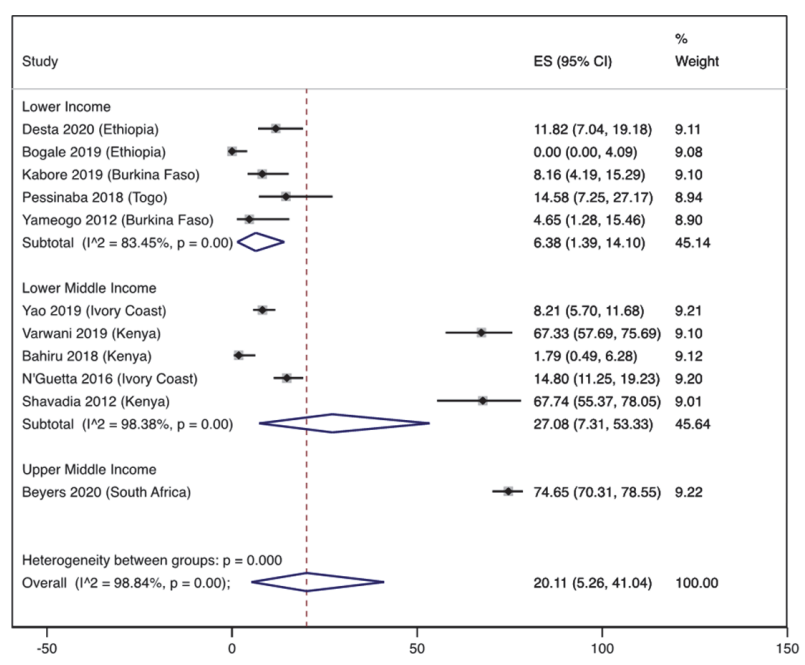

Abstract 43 Figure 2 Meto-anal ysis of the proportion of patients with STEMI who received emergency coronary reperfusion (either $\mathrm{PCl}$ or thrombolysis) stratified by country income level

presentation to hospital. This is the first meta-analysis attempting to pool the in-hospital mortality and emergency treatment of patients with STEMI in SSA. The studies included were of moderate to poor quality and suffered from small sample sizes. As the burden of IHD increases in SSA, health systems must adapt to be able to produce better outcomes for patients presenting with STEMI.

Conflict of Interest None

\section{IDENTIFYING LOW-RISK RISK CHEST PAIN IN THE EMERGENCY DEPARTMENT WITHOUT TROPONIN TESTING: A VALIDATION STUDY OF THE HE-MACS AND HEAR RISK SCORES}

Fraser Todd, James Duff, Ed Carlton. North Bristol Trust, Bristol, UK

10.1136/heartjnl-2021-BCS.44
Introduction Patients presenting to Emergency Departments with chest pain of possible cardiac origin represent a substantial and challenging cohort to risk stratify. Scores such as HEMACS (History and Electrocardiogram-only Manchester Acute Coronary Syndromes decision aid) and HEAR (History, ECG, Age, Risk factors) have been developed to stratify risk without the need for troponin testing. Validation of these scores remains limited at present.

Methods We performed a post-hoc analysis of the LoDED (Limit of Detection and ECG discharge strategy) randomisedcontrolled trial dataset. Data collected during the trial, and prior to troponin results, were used to calculate HEAR and HE-MACS scores. Previously published thresholds of $<4 \%$ for HE-MACS and $<2$ for HEAR defined very low risk patients. The primary outcome of MACE (major adverse cardiac events) at 30 days was used to assess diagnostic accuracy.

Results 629 patients were included, with a 7\% (42/629) incidence of MACE within 30 days. HE-MACS and HEAR scores identified $85 / 629$ and $181 / 629$ patients predicted to be at very low risk of MACE. Within these cohorts, MACE occurred in $0 / 85$ and $1 / 181$ patients respectively. Sensitivity of HE-MACS and HEAR were 100\% (95\% CI: 91.6-100\%) and 97.6\% (95\% CI: 87.7-99.9\%) respectively. Receiver operating characteristic (ROC) curves demonstrated area under curve (AUC) of 0.80 [95\%CI: 0.74-0.85] for HE-MACS and 0.76 [95\% CI: 0.69-0.82] for HEAR.

Conclusion HEAR and HE-MACS show potential as rule out tools for acute myocardial infarction without the need for troponin testing. However, prospective studies are required to further validate these scores for clinical implementation.

Conflict of Interest None declared

\section{COMPLETE REVASCULARISATION IS ASSOCIATED WITH IMPROVED SURVIVAL AFTER OUT OF HOSPITAL CARDIAC ARREST}

Ritesh Kanyal, Nilesh Pareek, Dhruv Sarma, Apurva Bharucha, Rafal Dworakowski, Narbeh Melikian, Ian Webb, Ajay Shah, Philip MacCarthy, Jonathan Byrne. King's College Hospital, London, UK

\subsection{6/heartjnl-2021-BCS.45}

Introduction Coronary artery disease (CAD) is common in patients with Out of Hospital Cardiac Arrest (OOHCA) but the clinical relevance of burden of $\mathrm{CAD}$ and evidence for revascularisation strategies in relation to outcomes and modes of death remains unclear. This study sought to assess the extent of CAD as defined by the SYNTAX score and prognostic value of complete compared with incomplete revascularisation by evaluating the SYNTAX revascularisation index (SRI) in patients with OOHCA.

Methods 619 patients with OOHCA were admitted at our centre between 1st May 2012 and 31st December 2017. 237 were excluded for having a non-cardiac aetiology or prior neurological disability. 398 patients were included into the study and of these 272 (xx\%) had early coronary angiography and were included in the final analysis. The baseline SYNTAX score (bSS) and residual SYNTAX score (rSS) were determined from the coronary angiograms by a blinded cardiologist. Patients were subdivided into 4 subgroups according to quartiles of the baseline syntax score (bSS) of 0, Group A: 1-10, Group B: $11-20$ and Group C: $\geq 21$. Complete 


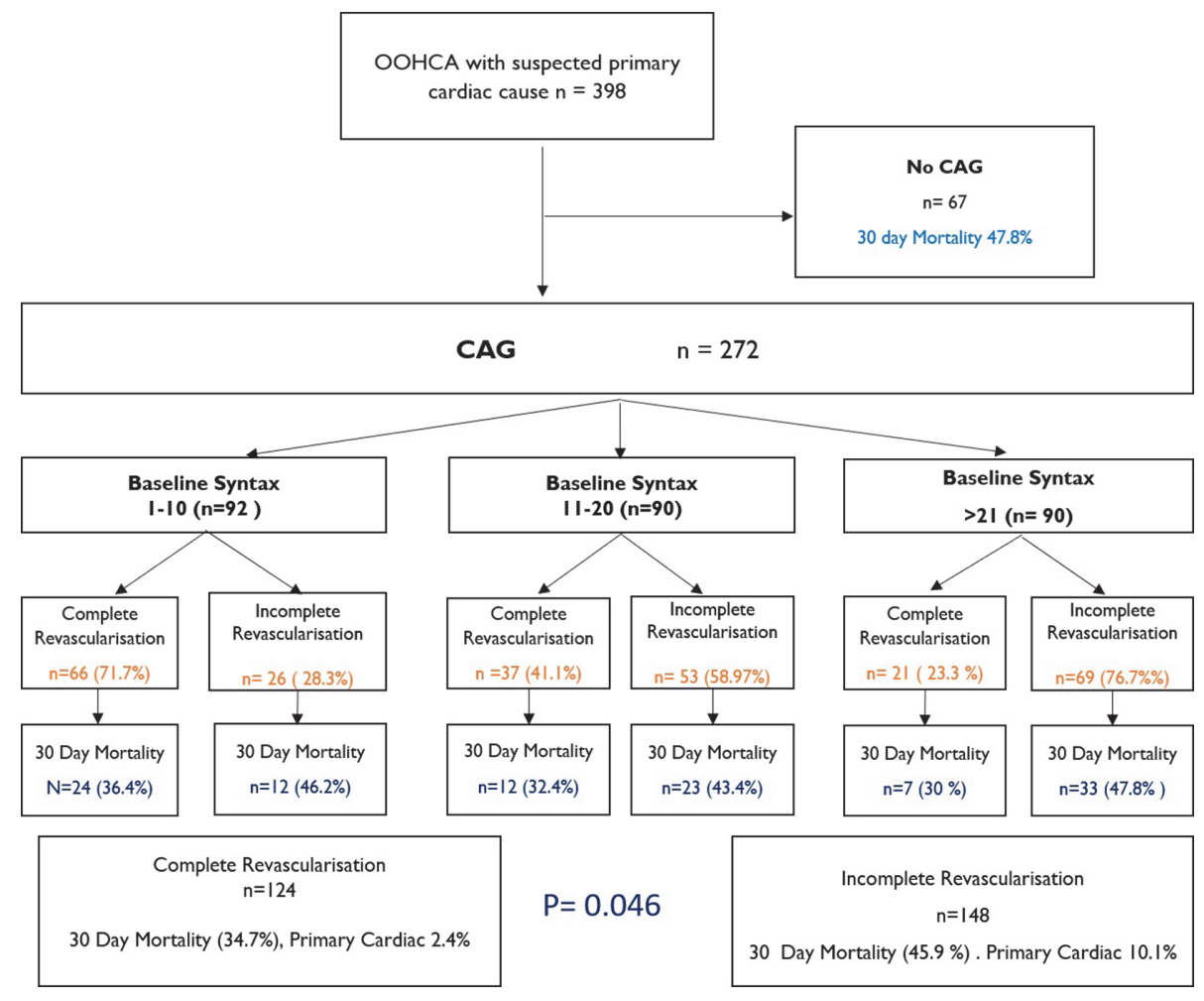

\section{Abstract 45 Figure 1}

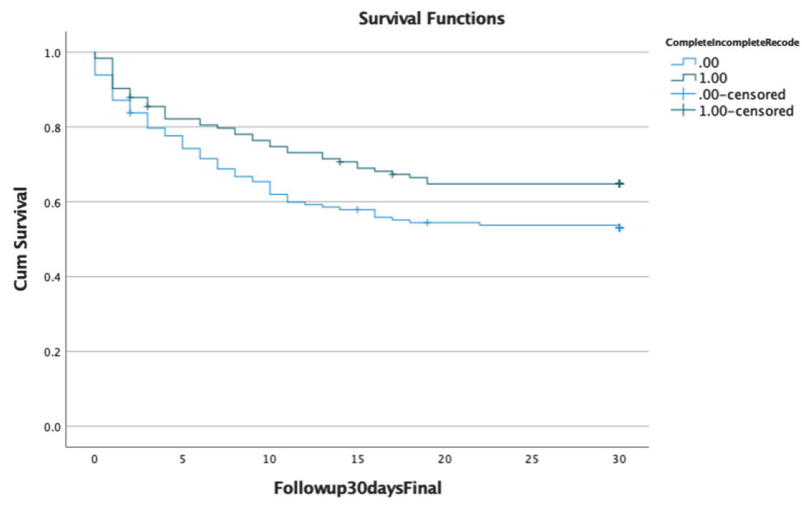

Abstract 45 Figure 2

revascularisation was defined as SRI of 1 and incomplete as SRI $<1$ where the SRI $=(1-[\mathrm{rSS} / \mathrm{bSS}]) \times 100$ (figure 1$)$.

Results Patients with a bss of 0 were younger, more likely to be female with, had less shockable initial arrest rhythms and worse lactate and $\mathrm{pH}$ on arrival than those with bss $>0$. Patients with bss $>0$ (i.e. those with coronary artery disease) had similar cardiac arrest circumstances in terms of rates of witnessed, bystander CPR and shockable rhythms. Admission metabolic status reflected by $\mathrm{pH}$ and lactate and rates of ST elevation/LBBB were also identical for all three bss groups. However, LVEF on admission decreased significantly as coronary complexity increased $(\mathrm{P}<0.0001)$. While early angiography was more preferentially performed in those with higher coronary complexity, paradoxically, those with bss 1-4 had highest rates of culprit lesions which was reflected in higher rates of PCI (table 1). Of the 273 patients who had PCI, 125 (45.8\%) had complete revascularisation (CR) compared with $54.2 \%$ with incomplete revascularisation (IR). Complete
Abstract 45 Table 1 Baseline Characteristics based on Baseline Syntax Scores

\begin{tabular}{|c|c|c|c|c|}
\hline & $\begin{array}{l}\text { bSS }(1-10) \\
n=92\end{array}$ & $\begin{array}{l}\text { bSS }(11-20) \\
n=90\end{array}$ & $\begin{array}{l}\text { bSS }>21 \\
n=90\end{array}$ & $p$ \\
\hline Age & 60.413 .3 & 62.812 .0 & 67.511 .6 & 0.003 \\
\hline Male (\%) & $71(77.1 \%)$ & $68(75.6 \%)$ & 77 (85.6\%) & 0.204 \\
\hline HTN (\%) & $42(45.7 \%)$ & $41(45.6 \%)$ & $46(51.1 \%)$ & 0.693 \\
\hline Diabetes (\%) & $13(14.1 \%)$ & $22(24.4 \%)$ & $13(14.4 \%)$ & 0.118 \\
\hline Previous CABG & $6(6.5 \%)$ & $10(11.1 \%)$ & $12(13.3 \%)$ & 0.304 \\
\hline Witnessed & $73(83.7 \%)$ & $76(84.4 \%)$ & $77(85.5 \%)$ & 0.491 \\
\hline Bystander CPR & $69(76.7 \%)$ & $69(75.7 \%)$ & $71(78.9 \%)$ & 0.823 \\
\hline Downtime (mins) & 25.018 .4 & 2814.5 & 2820.8 & 0.516 \\
\hline VF/VT & $73(79.3 \%)$ & $74(82.2 \%)$ & $75(83.3 \%)$ & 0.773 \\
\hline Epinephrine use & $58(63.0 \%)$ & $58(64.4 \%)$ & $56(62.2 \%)$ & 0.978 \\
\hline $\mathrm{pH}$ & $7.23(7.14-7.30)$ & $7.25(7.10-7.34)$ & $7.25(7.12-7.33)$ & 0.513 \\
\hline Lactate & $4.2(2 .-8.3)$ & $3.5(2.0-7.1)$ & $3.8(2.0-8.0)$ & 0.737 \\
\hline STEMI/LBBB & $58(63.0 \%)$ & $69(76.7 \%)$ & $69(76.7 \%)$ & \\
\hline LVEF (\%) & 459.3 & 4010.0 & 359.3 & $<0.0001$ \\
\hline Early angiography & $77(83.6 \%)$ & $84(93 \%)$ & $84(93 \%)$ & 0.042 \\
\hline Culprit lesion & $71 / 77(92.2 \%)$ & $73 / 84(86.9 \%)$ & $63 / 84(75.0 \%)$ & 0.008 \\
\hline $\mathrm{PCl}$ & $68 / 77(88.3 \%)$ & $71 / 84(84.5 \%)$ & $58 / 84(69.0 \%)$ & 0.004 \\
\hline SCAI C-E & $39 / 91(43.9 \%)$ & $41 / 90(45.6 \%)$ & $49 / 89(55.1 \%)$ & $\mathrm{p}=\mathrm{ns}$ \\
\hline
\end{tabular}

revascularisation was most likely to be achieved in as the coronary complexity reduced (Group A-10-71.1\%, Group B $41.1 \%$, Group C- 23.3\%). There was no difference in rates of cardiogenic shock between both groups (CR 61.1\% vs. IR $69 \% \mathrm{p}=\mathrm{ns})$, but patients with complete revascularisation were younger $(58.8$ vs $67.8, \mathrm{p}<0.0001)$ with lower rates of hypertension previous coronary artery bypass graft (CABG) $(16.2 \%$ vs $3.2 \%, \mathrm{p} \leq 0.0001)$. Admission metabolic status was identical 
Abstract 45 Table 2 Baseline characteristics of Incomplete vs. Complete Revascularisation

\begin{tabular}{|c|c|c|c|}
\hline & $\begin{array}{l}\text { Incomplete } \\
\text { Revascularisation } \\
n=148\end{array}$ & $\begin{array}{l}\text { Complete } \\
\text { Revascularisation } \\
(n=124)\end{array}$ & $\mathrm{p}$ \\
\hline Age & $67.8 \pm 11.1$ & $58.8 \pm 12.7$ & $<0.0001$ \\
\hline Male (\%) & $119(80.4 \%)$ & $97(77.6 \%)$ & 0.570 \\
\hline HTN (\%) & $82(55.4 \%)$ & $48(38.4 \%)$ & 0.005 \\
\hline Diabetes (\%) & $31(20.9 \%)$ & $17(13.6 \%)$ & 0.112 \\
\hline Previous CABG & $24(16.2 \%)$ & $4(3.2 \%)$ & $<0.0001$ \\
\hline Witnessed & $124(83.8 \%)$ & $103(82.4 \%)$ & 0.761 \\
\hline Bystander CPR & $112(75.7 \%)$ & $98(78.4 \%)$ & 0.595 \\
\hline Downtime (mins) & $28 \pm 19.2$ & $25.0 \pm 19.1$ & 0.406 \\
\hline VF/VT & $117(79.1 \%)$ & $106(84.8 \%)$ & 0.221 \\
\hline Epinephrine use & $98(66.2 \%)$ & $75(60.0 \%)$ & 0.371 \\
\hline $\mathrm{pH}$ & $7.24(6.78-7.52)$ & $7.24(6.35-7.47)$ & 0.433 \\
\hline Lactate & $3.9(1-16)$ & $4.0(1-26)$ & 0.585 \\
\hline STEMI/LBBB & $106(71.6 \%)$ & $91(72.8 \%)$ & \\
\hline LVEF (\%) & $40 \pm 10.3$ & $40.0 \pm 9.2$ & 0.13 \\
\hline Early angiography & $136 / 148(91.9 \%)$ & $110 / 125(88.0 \%)$ & 0.283 \\
\hline Culprit lesion & $98 / 136(72.1 \%)$ & $109 / 110(99.1 \%)$ & $<0.0001$ \\
\hline $\mathrm{PCl}$ & $89 / 136(65.4 \%)$ & $109 / 110(99.1 \%)$ & $<0.0001$ \\
\hline SYNTAX score & $19.8(1-53)$ & $10.0(2.0-38.5)$ & $<0.0001$ \\
\hline$C-E$ & 78/147 (53.1\%) & $50 / 123(40.7 \%)$ & 0.331 \\
\hline Final Primary & $80 / 148(54.1 \%)$ & $55 / 125(44 \%)$ & 0.098 \\
\hline \multicolumn{4}{|l|}{ Endpoint } \\
\hline 30 day Mortality & $68 / 148(45.9 \%)$ & $43 / 125(34.4 \%)$ & 0.049 \\
\hline $\begin{array}{l}\text { Cardiac death at } 30 \\
\text { days }\end{array}$ & $22 \%$ & $7 \%$ & 0.1 \\
\hline
\end{tabular}

between both groups.Complete revascularisation was associated with decreased mortality at 30 days $(45.9 \%$ vs $34.4 \%$, p $=0.049)$ and 12 months $(49.3 \%$ vs $35.2 \%, p=0.022)$. The lower mortality rate in CR was driven by lower cardiac deaths but this was not statistically significant $(22 \%$ vs $7 \%, \mathrm{p}=0.1)$ (figure 2).
Conclusions Complete revascularisation in a primary coronary aetiology OOHCA group is associated with reduced early and long-term mortality, which may be driven by a reduction in cardiac deaths. Prospective randomised trials in this population are warranted.

Conflict of Interest None

\section{THE RELATIONSHIP BETWEEN HIGH-SENSITIVITY TROPONIN TAKEN ON ADMISSION TO CRITICAL CARE, REGARDLESS OF WHETHER THERE WAS A CLINICAL INDICATION FOR TESTING, AND ONE YEAR MORTALITY: A NOVEL BIOMARKER FOR OUTCOME?}

'Jonathan Hinton, 'Maclyn Augustine, 'Lavinia Gabara, ${ }^{1}$ Mark Mariathas, ${ }^{1}$ Rick Allan, ${ }^{1}$ Florina Borca, ${ }^{1}$ Zoe Nicholas, ${ }^{1}$ Neil Gillett, ${ }^{2}$ Chun Shing Kwok, ${ }^{1}$ Paul Cook, ${ }^{3}$ Michael Grocott, ${ }^{2}$ Mamas Mamas, ${ }^{3}$ Nick Curzen. ${ }^{1}$ University Hospital Southampton, Southampton, UK; ${ }^{2}$ University of Keele; ${ }^{3}$ University of Southampton

\subsection{6/heartjnl-2021-BCS.46}

Introduction High-sensitivity troponin (hs-cTn) assays now form a key component of the diagnostic pathways for patients presenting to emergency medical services with chest pain. However, hs-cTn concentrations above the manufacturer-provided upper limit of normal (ULN) are now frequently reported in patients presenting with conditions not traditionally associated with type 1 myocardial infarction (T1MI). This is particularly true of severe illness states. We investigated the possible association between hs-cTn and one year mortality in critical care patients.

Method Consecutive patients admitted to two adult critical care units (general critical care unit (GCCU) and neuroscience critical care unit (NCCU)) over a six month period had hscTnI assay performed on admission, regardless of whether there was a clinical indication, and the results nested unless a clinical request had been made. Comorbidity data, illness severity and critical care outcome were recorded and have been previously reported. One year mortality data were obtained from NHS Digital.

Results After excluding patients diagnosed with T1MI by the clinical team, there were 1,033 patients remaining. At one

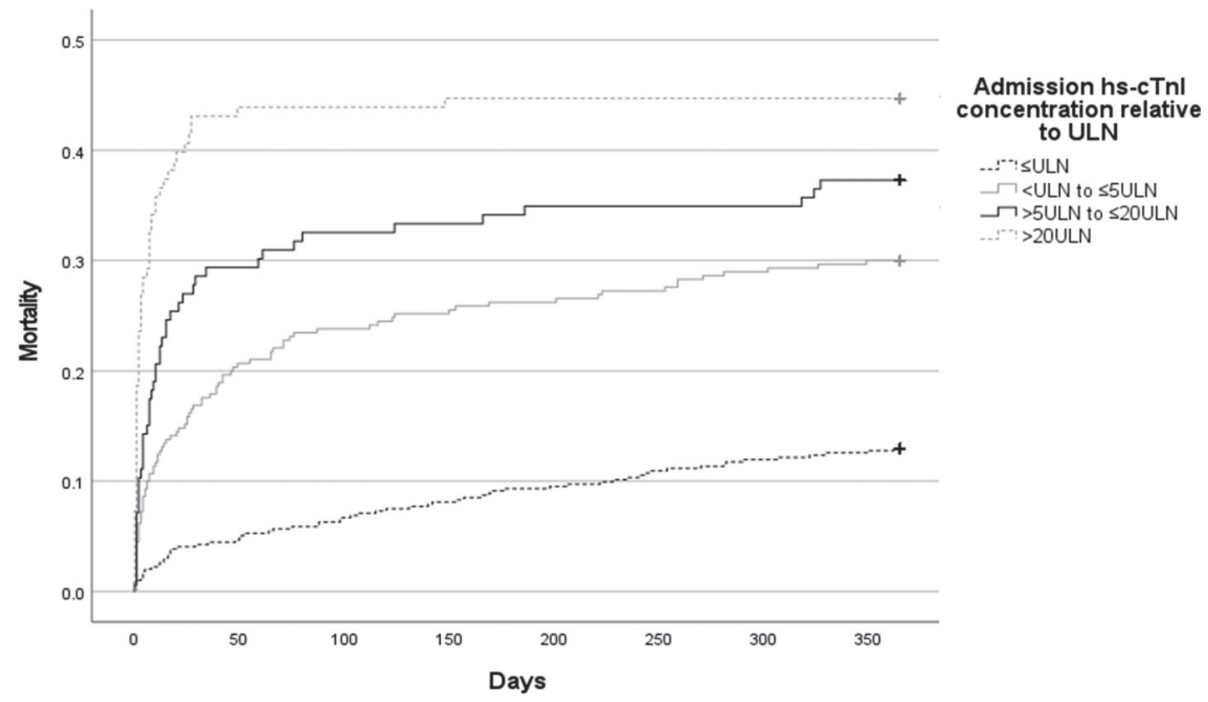

Abstract 46 Figure 1 Kaplan-Meier curve of one year mortality for increasing admission hs-cTnl concentrations relative to the ULN 